\title{
Stiff competition
}

ARISING FROM J. B. Berger, H. N. G. Wadley \& R. M. McMeeking, Nature 543, 533-537 (2017); https://doi.org/10.1038/nature21075

The paper of Berger, Wadley \& McMeeking ${ }^{1}$ presents beautiful results on structured composites near the edge of maximal stiffness for a given porosity. However, it appears that the authors were unaware of the large body of work on this subject, much of it summarized in refs ${ }^{2-6}$. In particular, their claim that "a material geometry that achieves the theoretical upper bounds for isotropic elasticity and strain energy storage (the Hashin-Shtrikman upper bounds) has yet to be identified" is not accurate. Multiscale elastically isotropic composites with simultaneously maximal bulk and shear modulus - and hence maximal stiffness and energy storage-were identified independently in refs ${ }^{7-9}$. There is a Reply to this Comment by J. B. Berger et al., Nature 564, https://doi. org/10.1038/s41586-018-0725-7 (2018).

Moreover, the simple argument made in ref. ${ }^{8}$ - that the HashinShtrikman bounds are attained if the actual field in the material matches the trial field, which is constant in one phase-shows that any hierarchical laminate, in which layers of the stiffer phase are sequentially added to the composite in different orientations, necessarily achieves these upper bounds if layering is done so that the final material is elastically isotropic. Later it was established by Bourdin and $\mathrm{Kohn}^{10}$ that no separation of length scales is needed if the volume fraction of the stiffer phase is small. These geometries are formed by the union of families of parallel plates, with each family having a different orientation, and include the cubic foam, octet foam and cubic + octet foam described in ref. ${ }^{1}$. The novelty of ref. ${ }^{1}$ is that it shows that this class of microstructure also works well if the volume fraction is moderate. Other porous three-dimensional microgeometries with very large bulk and shear moduli, at a moderate volume fraction of 0.338 , have been found using topology optimization methods ${ }^{11}$ (see, in particular, point e in figure 9 of ref. ${ }^{11}$ ). Yet it is still not known if a single-scale geometry can exactly attain the shear bounds away from the low-density limit. Single-scale geometries can achieve the bulk modulus bounds ${ }^{12-14}$.

To finish, we briefly mention important results that cover more general questions than those addressed in ref. ${ }^{1}$ to bring readers up to speed on current developments. If the second material is not void, there are improved bounds that couple the possible bulk and shear moduli ${ }^{15,16}$, and the range of possible (bulk, shear) pairs has been explored numerically ${ }^{11,17}$. A recent paper $^{18}$ goes a long way to completely characterizing the possible elasticity tensors of threedimensional printed, possibly anisotropic, materials constructed from a given isotropic material with given porosity. These materials include elastically isotropic microstructures that asymptotically attain the Hashin-Shtrikman upper bulk modulus bound for any given volume fraction, yet have an arbitrarily small shear modulus. If one allows the starting material to be as stiff as one likes, and replaces the void material by a material that is as compliant as one likes, then one can get any desired elasticity tensor ${ }^{19}$-a result also suggested by numerics ${ }^{17}$. In fact, non-local effective behaviours are possible too and, remarkably, these have also been completely characterized for linear elasticity ${ }^{20}$. In principle, one can obtain composites for which uniform strains cost little energy, but gradients in the strains (double gradients of the displacement) cost considerable energy (see, for example, ref. ${ }^{21}$ for some interesting examples).

\section{Data availability}

All data are available from the corresponding author upon reasonable request.

\section{G. W. Milton ${ }^{1} *$}

${ }^{1}$ Department of Mathematics, The University of Utah, Salt Lake City, UT, USA. *e-mail: milton@math.utah.edu

Received: 2 February; Accepted: 12 September 2018; Published online 5 December 2018.

1. Berger, J. B., Wadley, H. N. G. \& McMeeking, R. M. Mechanical metamaterials at the theoretical limit of isotropic elastic stiffness. Nature 543, 533-537 (2017).

2. Cherkaev, A. Variational Methods for Structural Optimization (Springer-Verlag, New York, 2000).

3. Milton, G. W. The Theory of Composites (Cambridge Univ. Press, Cambridge, 2002).

4. Allaire, G. Shape Optimization by the Homogenization Method (Springer-Verlag, New York, 2012).

5. Torquato, S. Random Heterogeneous Materials: Microstructure and Macroscopic Properties (Springer Science \& Business Media, New York, 2002).

6. Tartar, L. The General Theory of Homogenization: A Personalized Introduction, (Springer-Verlag, Berlin, Heidelberg, 2009)

7. Norris, A. N. A differential scheme for the effective moduli of composites. Mech. Mater. 4, 1-16 (1985).

8. Milton, G. W. in Homogenization and Effective Moduli of Materials and Media (eds Ericksen, J. L. et al.) 150-174 (Springer-Verlag, New York, 1986).

9. Francfort, G. A. \& Murat, F. Homogenization and optimal bounds in linear elasticity. Arch. Ration. Mech. Anal. 94, 307-334 (1986).

10. Bourdin, B. \& Kohn, R. V. Optimization of structural topology in the highporosity regime. J. Mech. Phys. Solids 56, 1043-1064 (2008).

11. Andreassen, E., Lazarov, B. S. \& Sigmund, O. Design of manufacturable 3D extremal elastic microstructure. Mech. Mater. 69, 1-10 (2014).

12. Vigdergauz, S. B. Effective elastic parameters of a plate with a regular system of equal-strength holes (Effektivnye uprugie parametry plastiny s reguliarnoi sistemoi ravnoprochnykh otverstii). Inzh. Zh. Mekh. Tver. Tela. 21, 165-169 (1986)

13. Grabovsky, Y. \& Kohn, R. V. Microstructures minimizing the energy of a two phase elastic composite in two space dimensions. II. The Vigdergauz microstructure. J. Mech. Phys. Solids 43, 949-972 (1995).

14. Liu, L., James, R. D. \& Leo, P. H. Periodic inclusion-matrix microstructures with constant field inclusions. Metall. Mater. Trans. A 38, 781-787 (2007).

15. Berryman, J. G. \& Milton, G. W. Microgeometry of random composites and porous media. J. Phys. D 21, 87-94 (1988)

16. Cherkaev, A. V. \& Gibiansky, L. V. Coupled estimates for the bulk and shear moduli of a two-dimensional isotropic elastic composite. J. Mech. Phys. Solids 41, 937-980 (1993).

17. Sigmund, O. Materials with prescribed constitutive parameters: an inverse homogenization problem. Int. J. Solids Struct. 31, 2313-2329 (1994).

18. Milton, G. W., Briane, M. \& Harutyunyan, D. On the possible effective elasticity tensors of 2-dimensional and 3-dimensional printed materials. Math. Mech. Complex Syst. 5, 41-94 (2017).

19. Milton, G. W. \& Cherkaev, A. V. Which elasticity tensors are realizable? J. Eng. Mater. Technol. 117, 483-493 (1995).

20. Camar-Eddine, M. \& Seppecher, P. Determination of the closure of the set of elasticity functionals. Arch. Ration. Mech. Anal. 170, 211-245 (2003).

21. Seppecher, P., Alibert, J.-J. \& dell'Isola, F. Linear elastic trusses leading to continua with exotic mechanical interactions. J. Phys. 319, 012018 (2011).

Competing interests Declared none.

\section{Additional information}

Reprints and permissions information is available at http://www.nature.com/ reprints.

Correspondence and requests for materials should be addressed to G.W.M.

https://doi.org/10.1038/s41586-018-0724-8 


\section{Berger et al. reply}

REPLYING TO G. W. Milton, Nature 564, https://doi.org/10.1038/s41586-018-0724-8 (2018)

In the accompanying Comment ${ }^{1}$, Milton correctly points out that material geometries that achieve the Hashin-Shtrikman upper bounds ${ }^{2}$ have been previously identified. We thank G. W. Milton for his interest in our work ${ }^{3}$ and welcome his insights, including those that look beyond our contribution in regard to elasticities that are realizable.

Milton correctly refutes our statement that "a material geometry that achieves the theoretical upper bounds for isotropic elasticity and strain energy storage (the Hashin-Shtrikman upper bounds ${ }^{2}$ ) has yet to be identified". In his Comment ${ }^{1}$, Milton has pointed to many studies that have identified material combinations and multi-length-scale geometries that achieve the Hashin-Shtrikman ${ }^{2}$ theoretical upper bound ${ }^{4-6}$. We acknowledge and accept this correction of our claim.

Retrospectively, it is clear that we should have qualified our claim and placed it in the narrower context of our study (namely, the design of a single-length-scale, single-material, elastically isotropic lattice that is easily fabricated) - a context motivated by the need for lightweighting and the continuing discovery of multifunctional structural systems. We did describe (to our knowledge, for the first time) a single-length-scale biphasic material geometry-specifically, a combination of void and solid phases - that performs at, or nearly at, the Hashin-Shtrikman upper bounds for both the bulk and shear moduli simultaneously, over a wide range of relative lattice densities. This design is simple and manufacturable and was demonstrated to achieve, or nearly achieve, the Hashin-Shtrikman ${ }^{2}$ theoretical upper bounds. In addition, because it is composed of two anisotropic but maximally efficient sub-geometries, it enables the creation of multifunctional lightweight structures. In our paper ${ }^{3}$, we provided analytical proof of our design's maximal elastic performance, as well as numerical evidence of its optimal elastic performance over a wide range of relative densities. In this restricted 'single-length-scale, single-material' context, we assess our claim to be accurate, and accept that we were remiss in not stating this context more clearly.

The summarizing works ${ }^{7-10}$ cited by Milton present techniques for generating optimal material microstructures. However, none directly addresses the problem that we sought to solve. Milton states that material geometries that achieve the Hashin-Shtrikman upper bounds simultaneously have previously been identified ${ }^{4-6}$. Although this is true, we find that there are notable differences that clearly differentiate our work from these studies. These are perhaps most evident in the geometric simplicity of our design and its implications for the fabricability, and therefore the utility, of our design as an engineering material system.

Although Norris ${ }^{4}$ identified a microstructure that simultaneously achieves the Hashin-Shtrikman upper bounds, this solution consists of solid disks embedded in vacuum, which is impractical. Francfort and Murat ${ }^{6}$ proved mathematically that laminates that stack in three dimensions can also simultaneously achieve the Hashin-Shtrikman upper bounds. However, the authors specify that both phases are solid, so low-density, single-solid-material systems with void space, such as ours, are not accessible. In both cases, voided regions can be approximated as a very-low-density phase. However, the design of this porous phase is still an issue, which is essentially identical to the fundamental problem of identifying a single-length-scale maximally stiff isotropic material geometry. This only adds to the difficulty of the solution by requiring material geometries to be constructed at even smaller length scales.

Ranked laminates have previously been shown to simultaneously achieve the Hashin-Shtrikman upper bounds ${ }^{5}$. Such laminates rely on multiple length scales, and at each level the smaller-scale composites are assumed to be isotropic and effectively continuous. We purposely avoid such complexity in our approach, in an effort to achieve simple and therefore manufacturable geometries.

Bourdin and Kohn ${ }^{11}$ studied a family of material geometries that contain parallel planes of material and that would appear to contain the cubic and octet geometries. These materials were found to simultaneously achieve the Hashin-Shtrikman upper bounds in the low-density limit-an aspect paralleled in our work. The authors performed numerical calculations to obtain two-dimensional solutions but did not go on to generate three-dimensional designs-thus avoiding what might be the most practical application of ref. ${ }^{11}$. Our work does parallel ref. ${ }^{11}$ in the recognition that sheets of material are required for optimal performance. This insight, regretfully, appears to have been overlooked
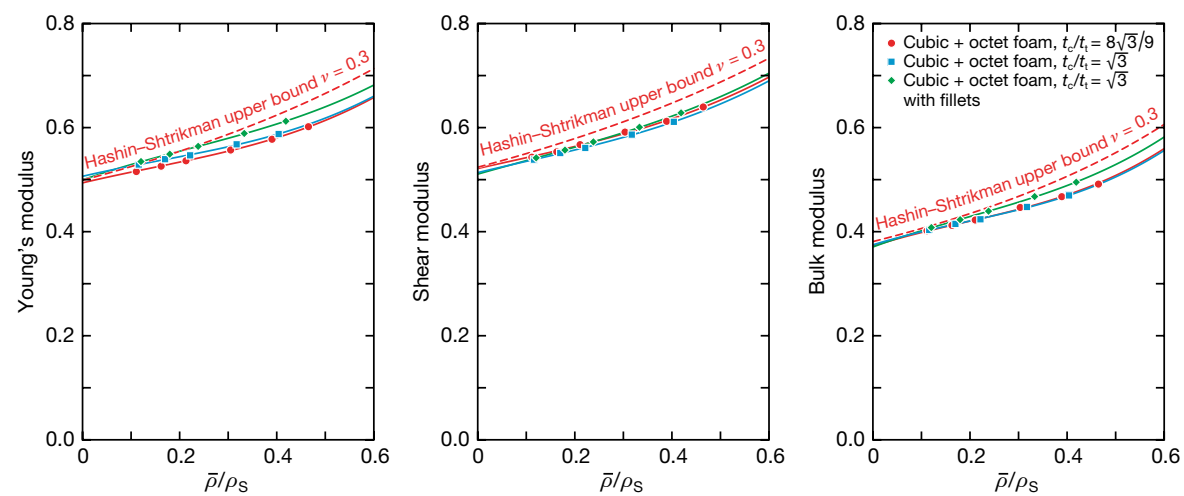

Fig. 1 | Young's, shear and bulk moduli. Finite-element analysis results indicate that with the addition of fillets, the normalized elastic moduli of the cubic + octet foam can achieve more than $98 \%$ of the theoretical upper limit for specific strain energy storage when the relative density is moderate to low, $\bar{\rho} / \rho_{\mathrm{S}} \leq 26.4 \%$; $\bar{\rho}$ is the effective density of the cellular material and $\rho_{\mathrm{S}}$ is the density of the constituent (solid) material ( $\nu$ is the
Poisson ratio). The filleted design with a wall thickness ratio of $t_{\mathrm{c}} / t_{\mathrm{t}}=\sqrt{3}$ at $\bar{\rho} / \rho_{\mathrm{S}}=26.4 \%$ has a shear performance that reaches $96.7 \%$ of the Hashin-Shtrikman upper limit. By varying the wall thickness ratio, isotropy can be achieved independently of the total performance, so that $98 \%$ of the shear upper bound can be realized at this relative density. 


\section{BRIEF COMMUNICATIONS ARISING}

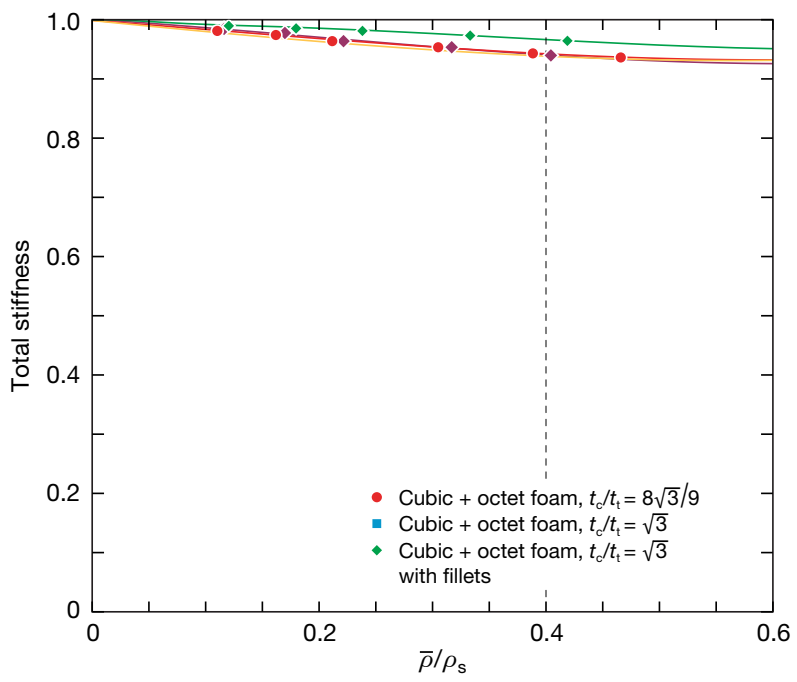

Fig. 2 Total stiffness. The total specific strain energy storage of the cubic + octet foam is enhanced by the addition of fillets. This topology achieves more than $96.6 \%$ of the upper bounds when $\bar{\rho} / \rho_{\mathrm{S}} \leq 40.0 \%$.

by the broader community, and we are unaware of any subsequent study that utilized it as a design principle to identify a manufacturable three-dimensional solution such as ours.

There are numerous studies that involve mathematical approaches to achieve extremal performance in composite systems. One of the primary issues with the further development of these systems into structural materials is the often-complex nature of these designs. For

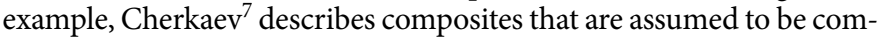
posed of small fragments, which are necessarily much smaller than the domain under consideration, with ranked laminates being a subset of these materials. The practicality of fabricating such complex geometries is questionable, since Cherkaev himself suggests that these are most useful as design guidelines for more practical approaches ${ }^{7}$.

There is certainly a large and interesting body of work in the area of topology and geometry optimization that address a space that encompasses and goes beyond the scope of our work-some of which Milton highlights ${ }^{12-18}$. We appreciate his identification of the parallels between our work and this important area of study.

While it is still not known whether a material geometry exists that can achieve the Hashin-Shtrikman shear upper bound away from the low-density limit, the cubic + octet foam achieves $94.7 \%$ of the HashinShtrikman upper bound on shear modulus, and $95.2 \%$ of the bound on Young's modulus, at a moderate relative density of 0.338 , while having a Zener anisotropy ratio of 1.01. By reducing stress concentrations by rounding the joints where webs intersect (that is, with the addition of fillets), these can be increased to $96.4 \%, 98.1 \%$ and 1.02 , respectively (this is with a wall thickness ratio of $t_{\mathrm{c}} / t_{\mathrm{t}}=\sqrt{3}-\operatorname{not} t_{\mathrm{c}} / t_{\mathrm{t}}=8 \sqrt{3 / 9}$, which is isotropic in the low-density limit ${ }^{3} ; t_{\mathrm{c}}$ and $t_{\mathrm{t}}$ are the wall thicknesses of the cubic and octet sub-geometries, respectively) (Fig. 1). These can easily be made isotropic by varying the ratio of the wall thicknesses, $t_{\mathrm{c}} / t_{\mathrm{t}}$. This improvement is not the result of rigorous optimization, but rather a simple ad hoc approach that leaves room for potentially even better-performing designs. If the results of Andreassen et al. ${ }^{19}$ are indeed relevant and noteworthy, as Milton points out, then it pays to mention that the cubic + octet foam does, in essence, achieve the theoretical upper bounds for structural efficiency away from the low-density limit (Fig. 2), including that for shear modulus, and that the identification of such a material geometry is not a completely open problem.
In the papers discussed above we find no description or illustration of a simple three-dimensional, low-density geometry with a single length scale and fabricated using a single solid material, that achieves the Hashin-Shtrikman upper bounds on elastic moduli; that is, we find nothing similar to the geometry that we have developed. Although the work of Bourdin and Kohn ${ }^{11}$ does appear to facilitate the generation of our extremal design, the authors do not use their numerical scheme to solve any three-dimensional problems, and their proofs address only the low-density limit. We acknowledge that Professor Milton has provided a helpful summary of theoretical approaches that complement the approach that we have taken. However, considering the limited space available for the presentation of our study, the focus of our paper ${ }^{3}$ was to describe and discuss our design approach and the mechanical properties of the resulting topology. This focus determined the emphasis of the work that we presented and restricted the literature that we selected to cite.

\section{Data availability}

The datasets generated and analysed during the current study are available from the corresponding author on reasonable request.

\section{J. B. Berger ${ }^{1,2}$, H. N. G. Wadley ${ }^{3}$ \& R. M. McMeeking ${ }^{1,2,4,5}$}

${ }^{1}$ Materials Department, University of California, Santa Barbara, CA, USA. ${ }^{2}$ Department of Mechanical Engineering, University of California, Santa Barbara, CA, USA. ${ }^{3}$ Department of Materials Science and Engineering, School of Engineering and Applied Science, University of Virginia, Charlottesville, VA, USA. ${ }^{4}$ School of Engineering, University of Aberdeen, King's College, Aberdeen, UK. ${ }^{5}$ INM-Leibniz Institute for New Materials, Saarbrücken, Germany.*e-mail: berger@engineering.ucsb.edu

Published online 5 December 2018.

1. Milton, G. W., Nature 564, https://doi.org/10.1038/s41586-018-0724-8 (2018).

2. Hashin, Z. \& Shtrikman, S. A variational approach to the theory of the elastic behaviour of multiphase materials. J. Mech. Phys. Solids 11, 127-140 (1963).

3. Berger, J. B., Wadley, H. N. G. \& McMeeking, R. M. Mechanical metamaterials at the theoretical limit of isotropic elastic stiffness. Nature 543, 533-537 (2017).

4. Norris, A. N. A differential scheme for the effective moduli of composites. Mech. Mater. 4, 1-16 (1985).

5. Milton, G. W. in Homogenization and Effective Moduli of Materials and Media (eds Ericksen, J. L. et al.) 150-174 (Springer-Verlag, New York, 1986).

6. Francfort, G. A. \& Murat, F. Homogenization and optimal bounds in linear elasticity. Arch. Ration. Mech. Anal. 94, 307-334 (1986).

7. Cherkaev, A. Variational Methods for Structural Optimization (Springer-Verlag, New York, 2000).

8. Milton, G. W. The Theory of Composites (Cambridge Univ. Press, Cambridge, 2002).

9. Allaire, G. Shape Optimization by the Homogenization Method (Springer-Verlag, New York, 2012).

10. Torquato, S. Random Heterogeneous Materials: Microstructure and Macroscopic Properties (Springer Science \& Business Media, New York, 2002).

11. Bourdin, B. \& Kohn, R. V. Optimization of structural topology in the highporosity regime. J. Mech. Phys. Solids 56, 1043-1064 (2008).

12. Berryman, J. G. \& Milton, G. W. Microgeometry of random composites and porous media. J. Phys. D 21, 87-94 (1988).

13. Cherkaev, A. V. \& Gibiansky, L. V. Coupled estimates for the bulk and shear moduli of a two-dimensional isotropic elastic composite. J. Mech. Phys. Solids 41, 937-980 (1993).

14. Sigmund, O. Materials with prescribed constitutive parameters: an inverse homogenization problem. Int. J. Solids Struct. 31, 2313-2329 (1994).

15. Milton, G. W. \& Cherkaev, A. V. Which elasticity tensors are realizable? J. Eng. Mater. Technol. 117, 483-493 (1995).

16. Milton, G. W., Briane, M. \& Harutyunyan, D. On the possible effective elasticity tensors of 2-dimensional and 3-dimensional printed materials. Math. Mech. Complex Syst. 5, 41-94 (2017).

17. Camar-Eddine, M. \& Seppecher, P. Determination of the closure of the set of elasticity functionals. Arch. Ration. Mech. Anal. 170, 211-245 (2003).

18. Seppecher, P., Alibert, J.-J. \& dell'Isola, F. Linear elastic trusses leading to continua with exotic mechanical interactions. J. Phys. 319, 012018 (2011). 
19. Andreassen, E., Lazarov, B. S. \& Sigmund, O. Design of manufacturable 3D extremal elastic microstructure. Mech. Mater. 69, 1-10 (2014).

Author contributions J.B.B. created the ideas, conceived and designed the new material geometries and performed the structural analysis. R.M.M. developed the analytical models for the strain energy and moduli and, with H.N.G.W., contributed to refining the concepts, contextualizing the results and providing critiques and assessments.

Competing interests The material geometry identified in this work to

achieve the theoretical bounds in performance has been included in a Patent
Cooperation Treaty (PCT/US2015/010458) by Nama Development, LLC (DE), which is majority-owned by J.B.B.

Additional information

Reprints and permissions information is available at http://www.nature.com/ reprints.

Correspondence and requests for materials should be addressed to J.B.B.

Publisher's note: Springer Nature remains neutral with regard to jurisdictional claims in published maps and institutional affiliations.

https://doi.org/10.1038/s41586-018-0725-7 